\title{
Synchronization of Two Fractional-Order Chaotic Systems via Nonsingular Terminal Fuzzy Sliding Mode Control
}

\author{
Xiaona Song,, Shuai Song, ${ }^{1}$ Ines Tejado Balsera, ${ }^{2}$ Leipo Liu, ${ }^{1}$ and Lei Zhang ${ }^{3}$ \\ ${ }^{1}$ School of Information Engineering, Henan University of Science and Technology, Luoyang 471023, China \\ ${ }^{2}$ Industrial Engineering School, University of Extremadura, Badajoz, Spain \\ ${ }^{3}$ School of Electrical Engineering, Henan University of Science and Technology, Luoyang 471023, China \\ Correspondence should be addressed to Xiaona Song; xiaona_97@163.com
}

Received 23 February 2017; Accepted 6 July 2017; Published 17 August 2017

Academic Editor: Petko Petkov

Copyright (C) 2017 Xiaona Song et al. This is an open access article distributed under the Creative Commons Attribution License, which permits unrestricted use, distribution, and reproduction in any medium, provided the original work is properly cited.

\begin{abstract}
The synchronization of two fractional-order complex chaotic systems is discussed in this paper. The parameter uncertainty and external disturbance are included in the system model, and the synchronization of the considered chaotic systems is implemented based on the finite-time concept. First, a novel fractional-order nonsingular terminal sliding surface which is suitable for the considered fractional-order systems is proposed. It is proven that once the state trajectories of the system reach the proposed sliding surface they will converge to the origin within a given finite time. Second, in terms of the established nonsingular terminal sliding surface, combining the fuzzy control and the sliding mode control schemes, a novel robust single fuzzy sliding mode control law is introduced, which can force the closed-loop dynamic error system trajectories to reach the sliding surface over a finite time. Finally, using the fractional Lyapunov stability theorem, the stability of the proposed method is proven. The proposed method is implemented for synchronization of two fractional-order Genesio-Tesi chaotic systems with uncertain parameters and external disturbances to verify the effectiveness of the proposed fractional-order nonsingular terminal fuzzy sliding mode controller.
\end{abstract}

\section{Introduction}

Fractional calculus has received an enormous amount of research effort in recent years, because it allows one to describe the behavior of a real system more accurately and more adequately compared to the standard calculus [1-3]. Although fractional calculus is a more than 300-year-old mathematical tool, its application in physics and engineering, especially in modeling and control, began only recently; for example, microelectromechanical systems [4] and systems consisting of viscoelastic materials [5] can be described more accurately using fractional calculus. Applications of fractional-order control techniques in chaotic systems have also been presented. It has been shown that several fractional-order systems exhibit chaotic behavior, such as Lorenz [6], Liu [7], and hyperchaotic system [8]. Due to the ease of fractional chaotic systems' electronics implementation and the rapid development of the stability of fractional differential equations, fractional chaotic systems have attracted a great deal of attention [9]. Owing to the potential applications of fractional-order chaotic systems in secure communication and control processing [10], the study of chaos synchronization in fractional-order dynamic systems is receiving increasing attention $[6,11]$. Therefore, the analysis and control/synchronization of fractional-order dynamical chaotic systems are important in both theory and practice. Up till now, many control/synchronization methods, such as active control [12], active sliding mode control [13], adaptive-impulsive control [14], fuzzy adaptive control [15], and generalized projective synchronization [16] and the references therein, have been successfully applied to control/synchronize the chaos of fractional-order chaotic systems.

On the other hand, sliding mode control is well known for its good robustness against disturbances and parameter uncertainties [17]; therefore, it has been one of the most interesting topics of research, and many researchers have made great contributions to this field [18-20]. In recent years, control and synchronization of fractional-order chaotic systems using sliding mode control techniques have attracted 
the attention of many scholars. As a result, some sliding mode control methods have been used to control and/or synchronize fractional-order chaotic systems $[21,22]$. In [23], the sliding mode controller design for fractional-order chaotic system is discussed, and the designed control scheme ensures asymptotical stability of the uncertain fractionalorder chaotic systems with an external disturbance. A fractional-order sliding mode controller is designed for a novel fractional-order hyperchaotic system in [24]. Among the sliding mode control community, nonsingular terminal sliding mode control has been widely investigated, since it can achieve finite-time convergence without causing any singularity problem in the process of traditional terminal sliding mode control design [25]. References [26, 27] have developed nonsingular terminal sliding mode control for fractionalorder chaotic systems, and to remove the chattering of the designed method, a nonchatter sliding surface was proposed in [28].

However, most of the works in the references toward synchronization/control of fractional-order chaotic systems have been performed in terms of the Lyapunov stability of the closed-loop system. In practice, the finite-time stability of fractional-order systems is an important issue. It has been known that the finite-time control of nonlinear systems gives rise to a high-precision performance as well as the finitetime convergence to origin [27]. Therefore, many researchers have done valuable work on finite time stability analysis and control for fractional-order systems [29, 30]; particularly for fractional-order chaotic systems, solutions to finite-time stabilizing and synchronization problems have been reported in [31, 32] and [33], respectively.

Hence, the field that combines the finite-time stability and the nonsingular terminal sliding mode control has become very popular. Many researchers have made great contributions; for example, for integer-order chaotic systems with uncertain parameters or disturbances, the finite-time nonsingular terminal sliding mode controller was designed in $[34,35]$, and, furthermore, for fractional-order chaotic systems, a novel fractional-order terminal sliding mode controller was proposed in [27] based on finite-time scheme. However, to the best of our knowledge, there is little work in the literature on finite-time control for fractional-order chaotic systems which combines the nonsingular terminal sliding mode control and the fuzzy control theory, which has remained as an open and challenging problem to be solved in this paper.

Motivated by the above discussions, this paper proposes a new fractional-order nonsingular fuzzy sliding mode controller for robust synchronization of two fractional-order chaotic systems with uncertain parameters and external disturbances. After introducing a new fractional-order terminal sliding surface, its finite-time stability is proven. Then, in terms of fractional Lyapunov stability theory, a robust fuzzy sliding mode control law is derived to ensure the occurrence of the sliding motion in a finite time. Finally, an illustrative example is given to demonstrate the effectiveness of the proposed control technique.

The rest of this paper is organized as follows. In Section 2, some preliminaries of fractional calculus are given. Section 3 details the problem formulation. The design procedure of the proposed fractional-order nonsingular terminal fuzzy sliding mode approach is discussed in Section 4. The effectiveness of the proposed synchronization control method is demonstrated via numerical simulation in Section 5 , followed by the conclusions in Section 6.

\section{Preliminaries of Fractional Calculus}

There are three commonly used definitions of the fractionalorder differential operator, namely, Riemann-Liouville, Grünwald-Letnikov, and Caputo. In this paper, the Caputo derivative is chosen, the definition of which is given in the following.

Definition 1 (see [36]). The Caputo derivative of $f(t)$ with order $\alpha, m-1<\alpha \leq m$, is given by

$$
{ }_{0}^{C} D_{t}^{\alpha} f(t)=\frac{1}{\Gamma(m-\alpha)} \int_{0}^{t} \frac{f^{(m)}(\tau)}{(t-\tau)^{\alpha-m+1}} d \tau,
$$

where $\Gamma$ is the Gamma function.

Some common properties that are used for the stability analysis of fractional-order systems are listed below.

Property 1. The following equality holds for the Caputo derivative:

$$
{ }_{t_{0}}^{C} D_{t}^{\alpha}\left({ }_{t_{0}}^{C} D_{t}^{m} f(t)\right)={ }_{t_{0}}^{C} D_{t}^{\alpha+m} f(t),
$$

where $m=0,1,2, \ldots ; n-1<\alpha \leq n ; n \geq 1$ is an integer.

Property 2. For the Caputo derivative, the following equality holds:

$$
{ }_{t_{0}}^{C} D_{t t_{0}}^{\alpha} D_{t}^{C} f(t)={ }_{t_{0}}^{C} D_{t}^{\alpha-\beta} f(t),
$$

where $\alpha \geq \beta \geq 0$.

\section{Problem Statement}

Consider the following uncertain nonautonomous fractionalorder chaotic system:

$$
\begin{aligned}
D^{\alpha} x(t) & =\left[\begin{array}{l}
D^{\alpha} x_{1}(t) \\
D^{\alpha} x_{2}(t) \\
D^{\alpha} x_{3}(t)
\end{array}\right] \\
& =\left[\begin{array}{c}
x_{2} \\
x_{3} \\
f(x)+\Delta f(x)+d_{x}(t)+u(t)
\end{array}\right],
\end{aligned}
$$

where $\alpha \in(0,1)$ is the order of the system, $x(t)=\left[x_{1}\right.$, $\left.x_{2}, x_{3}\right]^{T} \in R^{3}$ is the state vector of the system, $f(x)$ is a given nonlinear function, which is dependent on the state vector $x$ and the time $t, \Delta f(x) \in R$ stands for the uncertain parameter 
term, $d_{x}(t) \in R$ represents the external disturbance, and $u(t) \in R$ is the control input, which will be designed later.

Now, define the chaos synchronization problem as follows: design an appropriate controller for system (4) such that the state trajectories of the above response system can track the state trajectories of the following drive chaotic system:

$$
D^{\alpha} y(t)=\left[\begin{array}{c}
D^{\alpha} y_{1}(t) \\
D^{\alpha} y_{2}(t) \\
D^{\alpha} y_{3}(t)
\end{array}\right]=\left[\begin{array}{c}
y_{2} \\
y_{3} \\
g(y)+\Delta g(y)+d_{y}(t)
\end{array}\right],
$$

where $y(t)=\left[y_{1}, y_{2}, y_{3}\right]^{T} \in R^{3}$ is the state vector of the system, $g(y)$ is a given nonlinear function of $y$ and $t, \Delta g(y) \epsilon$ $R$ is an unknown model uncertainty term, and $d_{y}(t) \in R$ stands for an external disturbance.

The error between the response system (4) and the drive system $(5)$ is $e(t)=y(t)-x(t)$, defined as

$$
e(t)=\left\{\begin{array}{l}
e_{1}(t)=y_{1}(t)-x_{1}(t) \\
e_{2}(t)=y_{2}(t)-x_{2}(t) \\
e_{3}(t)=y_{3}(t)-x_{3}(t)
\end{array}\right.
$$

and its fractional-order dynamics are represented as

$$
D^{\alpha} e(t)=\left\{\begin{array}{l}
D^{\alpha} e_{1}(t)=e_{2} \\
D^{\alpha} e_{2}(t)=e_{3} \\
D^{\alpha} e_{3}(t)=g(y)-f(x)+\Delta g(y)-\Delta f(x)+d_{y}(t)-d_{x}(t)-u(t) .
\end{array}\right.
$$

To design the following fuzzy sliding mode controller, we should transfer systems (4), (5), and (7) into the corresponding Takagi-Sugeno (T-S) fuzzy modes in terms of the T-S fuzzy modeling theory. Now, we give the following T-S fuzzy models, which are the reconstructed models of systems (4), (5), and (7).

Plant Rule $i$. If $z_{1}(t)$ is $F_{i 1}$ and $z_{2}(t)$ is $F_{i 2}$ and $z_{N}(t)$ is $F_{i N}$, then for the response system (4), the final output of the T-S fuzzy system is inferred as follows:

$$
\begin{aligned}
D^{\alpha} x(t)= & \sum_{i=1}^{N} h_{i}(z(t))\left[A_{i} x(t)\right]+\Delta \bar{f}(x)+\bar{d}_{x}(t) \\
& +U(t)
\end{aligned}
$$

where

$$
\begin{aligned}
h_{i}(z(t)) & =\frac{\omega_{i}(z(t))}{\sum_{i=1}^{N} \omega_{i}(z(t))}, \\
\omega_{i}(z(t))=\prod_{j=1}^{p} F_{i j}\left(z_{j}(t)\right), & \quad i=1,2, \ldots, N, \\
\omega_{i}(z(t)) \geq 0, & \\
\sum_{i=1}^{N} \omega_{i}(z(t))>0, & \\
h_{i}(z(t)) & \geq 0, \\
\sum_{i=1}^{N} h_{i}(z(t)) & =1,
\end{aligned}
$$

$x(t) \in R^{3}$ is the state vector of the system and $z_{1}(t), \ldots, z_{N}(t)$ are the premise variables. Throughout this paper, it is assumed that the premise variables do not depend on control variables and external disturbances, $F_{i j}\left(z_{j}(t)\right)$ is the grade of membership of $z_{j}(t)$ in $F_{i j}, A_{i} \in R^{3 \times 3}(i=1,2, \ldots, N)$ are known real constant matrices, $N$ is the number of IF-THEN rules, and

$$
\begin{gathered}
\Delta \bar{f}(x)=\left[\begin{array}{c}
0 \\
0 \\
\Delta f(x)
\end{array}\right], \\
\bar{d}_{x}(t)=\left[\begin{array}{c}
0 \\
0 \\
d_{x}(t)
\end{array}\right], \\
U(t)=\left[\begin{array}{c}
0 \\
0 \\
u(t)
\end{array}\right] .
\end{gathered}
$$

Using a similar process, drive system (5) and the error dynamic system (7) can be reconstructed to the following T-S fuzzy models:

$$
\begin{aligned}
D^{\alpha} y(t)= & \sum_{i=1}^{N} h_{i}(z(t))\left[\bar{A}_{i} y(t)\right]+\Delta \bar{g}(y)+\bar{d}_{y}(t), \\
D^{\alpha} e(t)= & \sum_{i=1}^{N} h_{i}(z(t))\left[\widehat{A}_{i} e(t)\right]+\Delta \bar{f}(x)-\Delta \bar{g}(y) \\
& +\bar{d}_{x}(t)-\bar{d}_{y}(t)-U(t),
\end{aligned}
$$


where $\bar{A}_{i} \in R^{3 \times 3}$ and $\widehat{A}_{i} \in R^{3 \times 3}$ are known real constant matrices and

$$
\begin{gathered}
\Delta \bar{g}(y)=\left[\begin{array}{c}
0 \\
0 \\
\Delta g(y)
\end{array}\right], \\
\bar{d}_{y}(t)=\left[\begin{array}{c}
0 \\
0 \\
d_{y}(t)
\end{array}\right] .
\end{gathered}
$$

For the above uncertain terms and external disturbances, we give the following assumptions.

Assumption 2 (see [35]). It is assumed that the uncertain terms $\Delta f(x)$ and $\Delta g(y)$ are bounded and the following inequality is satisfied:

$$
\left|D^{1-\alpha}(\Delta g(y)-\Delta f(x))\right| \leq L_{1},
$$

where $L_{1}$ is a known positive constant.

Assumption 3 (see [35]). It is assumed that the external disturbances $d_{x}(t)$ and $d_{y}(t)$ are bounded, and the following inequality is satisfied:

$$
\left|D^{1-\alpha}\left(d_{y}(t)-d_{x}(t)\right)\right| \leq L_{2},
$$

where $L_{2}$ is a given positive constant.

Definition 4. Consider the error dynamic system (12); if there exists a real number $T>0, \lim _{t \rightarrow T}\|e(t)\|=0$ is satisfied; meanwhile when $t>T,\|e(t)\| \equiv 0$. Then the state trajectories of the error dynamic system (12) will converge to zero in a finite time $T$.

The aim of this paper is to design a fractional-order nonsingular terminal fuzzy sliding mode controller to stabilize the error dynamic system (12). In other words, the goal of this paper is to synchronize the response system (4) and the drive system (5). In the next section, the controller design method will be given in detail.

\section{Fractional-Order Nonsingular Terminal Fuzzy Sliding Mode Controller Design}

In this section, firstly, a new fractional-order terminal sliding surface is introduced; then we propose proper sliding mode control laws to guarantee the existence of the sliding motion in a finite time.

To accomplish the sliding mode controller design, firstly, we propose a new fractional-order nonsingular terminal sliding surface as follows:

$$
\begin{aligned}
s(t) & \\
= & e_{2}+e_{3} \\
& +D^{\alpha-1}\left(k_{1} e_{1}+k_{2} e_{2}+k_{3} \operatorname{sgn}\left(e_{1}+e_{2}\right)\left|e_{1}+e_{2}\right|^{\theta}\right),
\end{aligned}
$$

where $0<\theta<1$ and $k_{1}, k_{2}$, and $k_{3}$ are positive scalars.
Once the system operates on the sliding mode, it satisfies the following equality:

$$
s(t)=0 .
$$

Using (16), the following sliding mode dynamics are obtained:

$$
\begin{aligned}
e_{2}+ & e_{3} \\
& +D^{\alpha-1}\left(k_{1} e_{1}+k_{2} e_{2}+k_{3} \operatorname{sgn}\left(e_{1}+e_{2}\right)\left|e_{1}+e_{2}\right|^{\theta}\right) \\
= & 0 .
\end{aligned}
$$

Using (7), it can be shown that (18) is equivalent to

$$
\begin{aligned}
& D^{\alpha} e_{1}+D^{\alpha} e_{2} \\
& \quad=-D^{\alpha-1}\left(k_{1} e_{1}+k_{2} e_{2}+k_{3} \operatorname{sgn}\left(e_{1}+e_{2}\right)\left|e_{1}+e_{2}\right|^{\theta}\right) .
\end{aligned}
$$

In the following theorem, the finite-time stability of the sliding mode dynamics (19) is proven.

Theorem 5. The sliding mode dynamics (19) are stable and their trajectories converge to the equilibrium point.

Proof. Select the following positive definite function as Lyapunov function candidates:

$$
V_{1}(t)=\left|e_{1}(t)+e_{2}(t)\right| .
$$

Taking the time derivative of $V_{1}(t)$, one can obtain

$$
\dot{V}_{1}(t)=\operatorname{sgn}\left(e_{1}+e_{2}\right)\left(\dot{e}_{1}(t)+\dot{e}_{2}(t)\right) .
$$

Based on Properties 1 and 2 and (21), we have

$$
\begin{gathered}
\dot{V}_{1}(t)=\operatorname{sgn}\left(e_{1}+e_{2}\right)\left(D^{1-\alpha}\left(D^{\alpha} e_{1}+D^{\alpha} e_{2}\right)\right) \\
=-\operatorname{sgn}\left(e_{1}+e_{2}\right) D^{1-\alpha}\left(D ^ { \alpha - 1 } \left(k_{1} e_{1}+k_{2} e_{2}\right.\right. \\
\left.\left.+k_{3} \operatorname{sgn}\left(e_{1}+e_{2}\right)\left|e_{1}+e_{2}\right|^{\theta}\right)\right)=-\operatorname{sgn}\left(e_{1}+e_{2}\right) \\
\cdot\left(k_{1} e_{1}+k_{2} e_{2}+k_{3} \operatorname{sgn}\left(e_{1}+e_{2}\right)\left|e_{1}+e_{2}\right|^{\theta}\right) . \\
\text { For } k_{1}=k_{2} \text {, the above equation gives } \\
\dot{V}_{1}(t)=-\left(k_{1}\left|e_{1}+e_{2}\right|+k_{3}\left|e_{1}+e_{2}\right|^{\theta}\right) \\
\quad \leq-k\left(\left|e_{1}+e_{2}\right|+\left|e_{1}+e_{2}\right|^{\theta}\right) \leq-k\left|e_{1}+e_{2}\right|<0,
\end{gathered}
$$

where

$$
k=\min \left\{k_{1}, k_{3}\right\} .
$$

From the above proof, we can conclude that the state trajectories of error dynamic system (7) will asymptotically converge to zero. Next, we prove that trajectories of error dynamic system (7) converge to zero in a finite time. 
From inequality (23), one has

$$
\dot{V}_{1}(t)=\frac{d\left|e_{1}+e_{2}\right|}{d t} \leq-k\left(\left|e_{1}+e_{2}\right|+\left|e_{1}+e_{2}\right|^{\theta}\right) .
$$

After simple calculations, one gets

$$
\begin{aligned}
d t & \leq-\frac{d\left|e_{1}+e_{2}\right|}{k\left(\left|e_{1}+e_{2}\right|+\left|e_{1}+e_{2}\right|^{\theta}\right)} \\
& =-\frac{d\left|e_{1}+e_{2}\right|^{1-\theta}}{k(1-\theta)\left(\left|e_{1}+e_{2}\right|^{1-\theta}+1\right)} .
\end{aligned}
$$

Integrating both sides of (26) from $t_{r}$ to $t_{s}$ and knowing that $x\left(t_{s}\right)=0$, one obtains

$$
\begin{aligned}
t_{s} & -t_{r} \leq-\frac{1}{k(1-\theta)} \int_{x\left(t_{r}\right)}^{x\left(t_{s}\right)} \frac{d\left|e_{1}+e_{2}\right|^{1-\theta}}{\left(\left|e_{1}+e_{2}\right|^{1-\theta}+1\right)} \\
& =-\frac{1}{k(1-\theta)} \ln \left(1+\left|e_{1}\left(x\left(t_{r}\right)\right)+e_{2}\left(x\left(t_{r}\right)\right)\right|^{1-\theta}\right) .
\end{aligned}
$$

Therefore, one can conclude that the error dynamics will converge to zero in a finite time $T \leq-(1 / k(1-\theta)) \ln (1+$ $\left.\left|e_{1}\left(x\left(t_{r}\right)\right)+e_{2}\left(x\left(t_{r}\right)\right)\right|^{1-\theta}\right)$.

A suitable nonsingular terminal sliding surface has been established in (16); the next step is to determine an input signal $u(t)$ to guarantee that the error system trajectories reach the sliding surface $s(t)=0$ and stay on it forever. When the closed-loop system is moving on the sliding surface, it satisfies the following equation:

$$
\dot{s}(t)=0 .
$$

Using (16), it follows that

$\dot{s}(t)$

$$
\begin{aligned}
= & \dot{e}_{2}+\dot{e}_{3} \\
& +D^{\alpha}\left(k_{1} e_{1}+k_{2} e_{2}+k_{3} \operatorname{sgn}\left(e_{1}+e_{2}\right)\left|e_{1}+e_{2}\right|^{\theta}\right) .
\end{aligned}
$$

Based on Properties 1 and 2, the following equation is obtained:

$$
\begin{aligned}
\dot{s}(t) & =D^{\alpha}\left(k_{1} e_{1}+k_{2} e_{2}+k_{3} \operatorname{sgn}\left(e_{1}+e_{2}\right)\left|e_{1}+e_{2}\right|^{\theta}\right) \\
& +D^{1-\alpha}\left[D^{\alpha} e_{2}\right]+D^{1-\alpha}\left[D^{\alpha} e_{3}\right]=D^{\alpha}\left(k_{1} e_{1}+k_{2} e_{2}\right. \\
& \left.+k_{3} \operatorname{sgn}\left(e_{1}+e_{2}\right)\left|e_{1}+e_{2}\right|^{\theta}\right) \\
& +D^{1-\alpha}\left[\sum_{i=1}^{2} h_{i}\left\{\sum_{j=1}^{3} \widehat{A}_{3 j} e_{j}\right\}+e_{3}+d_{y}(t)-d_{x}(t)\right. \\
& +\Delta g(y)-\Delta f(x)-u(t)] .
\end{aligned}
$$

If $\dot{s}(t)=0$, one has

$$
\begin{aligned}
u_{\mathrm{eq}} & =\sum_{i=1}^{2} h_{i}\left\{\sum_{j=1}^{3} \widehat{A}_{3 j} e_{j}\right\}+d_{y}(t)-d_{x}(t)+\Delta g(y) \\
& -\Delta f(x)+e_{3} \\
& +D^{2 \alpha-1}\left(k_{1} e_{1}+k_{2} e_{2}+k_{3} \operatorname{sgn}\left(e_{1}+e_{2}\right)\left|e_{1}+e_{2}\right|^{\theta}\right) .
\end{aligned}
$$

Then, select the following reaching law:

$$
u_{r}=D^{\alpha-1}\left(\xi_{1} s+\xi_{2}|s|^{\beta} \operatorname{sgn}(s)+\xi_{3}|s|^{\gamma} \operatorname{sgn}(s)\right)
$$

where $\xi_{1}, \xi_{2}$, and $\xi_{3}$ are the switching gains and positive constant scalars and $\beta, \gamma \in(0,1)$ are positive constant scalars.

Based on (31) and (32), the overall control $u(t)$ in the proposed control scheme is determined by

$U(t)$

$$
\begin{aligned}
= & \sum_{i=1}^{2} h_{i}\left\{\sum_{j=1}^{3} \widehat{A}_{3 j} e_{j}\right\}+d_{y}(t)-d_{x}(t)+\Delta g(y) \\
& -\Delta f(x)+e_{3} \\
& +D^{2 \alpha-1}\left(k_{1} e_{1}+k_{2} e_{2}+k_{3} \operatorname{sgn}\left(e_{1}+e_{2}\right)\left|e_{1}+e_{2}\right|^{\theta}\right) \\
& +D^{\alpha-1}\left(\xi_{1} s+\xi_{2}|s|^{\beta} \operatorname{sgn}(s)+\xi_{3}|s|^{\gamma} \operatorname{sgn}(s)\right) .
\end{aligned}
$$

Considering that the system uncertainties and external disturbances are unknown and unmeasurable, the proposed control input is modified as follows:

$$
\begin{aligned}
& u(t) \\
& =\sum_{i=1}^{2} h_{i}\left\{\sum_{j=1}^{3} \widehat{A}_{3 j} e_{j}\right\}+D^{\alpha-1}\left[\left(L_{1}+L_{2}\right) \operatorname{sgn}(s)\right]+e_{3} \\
& \quad+D^{2 \alpha-1}\left(k_{1} e_{1}+k_{2} e_{2}+k_{3} \operatorname{sgn}\left(e_{1}+e_{2}\right)\left|e_{1}+e_{2}\right|^{\theta}\right) \\
& \quad+D^{\alpha-1}\left(\xi_{1} s+\xi_{2}|s|^{\beta} \operatorname{sgn}(s)+\xi_{3}|s|^{\gamma} \operatorname{sgn}(s)\right) .
\end{aligned}
$$

The following theorem ensures that the error trajectories will converge to the sliding surface.

Theorem 6. Considering the error dynamic system (12), if this system is controlled by the control input (34), then the system trajectories will converge to the sliding surface $s(t)=0$ in a finite time.

Proof. Select a positive definite Lyapunov function as follows:

$$
V_{2}(t)=|s(t)| .
$$


Taking its time derivative, one has

$$
\dot{V}_{2}(t)=\operatorname{sgn}(s) \dot{s}(t) .
$$

Taking (30) into (36), one gets

$$
\begin{aligned}
& \dot{V}_{2}(t)=\operatorname{sgn}(s)\left\{D ^ { \alpha } \left(k_{1} e_{1}+k_{2} e_{2}\right.\right. \\
& \left.+k_{3} \operatorname{sgn}\left(e_{1}+e_{2}\right)\left|e_{1}+e_{2}\right|^{\theta}\right) \\
& +D^{1-\alpha}\left[\sum_{i=1}^{2} h_{i}\left\{\sum_{j=1}^{3} \widehat{A}_{3 j} e_{j}\right\}+e_{3}+d_{y}(t)-d_{x}(t)\right. \\
& +\Delta g(y)-\Delta f(x)-u(t)]\} .
\end{aligned}
$$

Based on inequalities (14), (15), and (34), one has

$$
\begin{aligned}
& \dot{V}_{2}(t) \leq L_{1}+L_{2}+\operatorname{sgn}(s) \\
& .\left\{D^{\alpha}\left(k_{1} e_{1}+k_{2} e_{2}+k_{3} \operatorname{sgn}\left(e_{1}+e_{2}\right)\left|e_{1}+e_{2}\right|^{\theta}\right)\right. \\
& \left.+D^{1-\alpha}\left[\sum_{i=1}^{2} h_{i}\left\{\sum_{j=1}^{3} \widehat{A}_{3 j} e_{j}\right\}+e_{3}-u(t)\right]\right\} .
\end{aligned}
$$

Using (34) and (38), it follows that

$$
\begin{aligned}
& \dot{V}_{2}(t) \leq \operatorname{sgn}(s)\left\{D ^ { \alpha } \left(k_{1} e_{1}+k_{2} e_{2}+k_{3} \operatorname{sgn}\left(e_{1}+e_{2}\right)\right.\right. \\
& \left.\cdot\left|e_{1}+e_{2}\right|^{\theta}\right)+D^{1-\alpha}\left[\sum_{i=1}^{2} h_{i}\left\{\sum_{j=1}^{3} \widehat{A}_{3 j} e_{j}\right\}+e_{3}\right. \\
& -\sum_{i=1}^{2} h_{i}\left\{\sum_{j=1}^{3} \widehat{A}_{3 j} e_{j}\right\}-e_{3}-D^{2 \alpha-1}\left(k_{1} e_{1}+k_{2} e_{2}\right. \\
& \left.\left.+k_{3} \operatorname{sgn}\left(e_{1}+e_{2}\right)\left|e_{1}+e_{2}\right|^{\theta}\right)\right]-\left(\xi_{1} s+\xi_{2}|s|^{\beta}\right. \\
& \left.\left.\cdot \operatorname{sgn}(s)+\xi_{3}|s|^{\gamma} \operatorname{sgn}(s)\right)\right\} .
\end{aligned}
$$

After some simple manipulations, one gets

$$
\begin{aligned}
& \dot{V}_{2}(t) \\
& \quad \leq \operatorname{sgn}(s)\left\{-\left(\xi_{1} s+\xi_{2}|s|^{\beta} \operatorname{sgn}(s)+\xi_{3}|s|^{\gamma} \operatorname{sgn}(s)\right)\right\} .
\end{aligned}
$$

Based on the equalities

$$
\begin{gathered}
\operatorname{sgn}(s) \cdot s=|s| \\
\operatorname{sgn}^{2}(s)=1,
\end{gathered}
$$

one gets

$$
\begin{aligned}
& \dot{V}_{2}(t) \\
& \quad \leq-\operatorname{sgn}(s)\left\{\xi_{1} s+\xi_{2}|s|^{\beta} \operatorname{sgn}(s)+\xi_{3}|s|^{\gamma} \operatorname{sgn}(s)\right\} \\
& \quad \leq-\left\{\xi_{1}|s|+\xi_{2}|s|^{\beta}+\xi_{3}|s|^{\gamma}\right\} \leq-\xi|s|,
\end{aligned}
$$

where

$$
\xi=\min \left\{\xi_{1}, \xi_{2}, \xi_{3}\right\}
$$

Therefore, based on Theorem 5, the state trajectories of the error dynamic system will converge to the sliding surface $s(t)=0$ in a finite time. To prove that the sliding motion occurs in finite time, one can obtain the reaching time as follows.

From inequalities (35) and (42), the following inequality can be obtained:

$$
\begin{aligned}
\dot{V}_{2}(t) & =\frac{d|s|}{d t} \leq-\xi\left(|s|+|s|^{\beta}+|s|^{\gamma}\right) \\
& \leq-\xi\left(|s|+|s|^{\beta}\right) .
\end{aligned}
$$

Setting $s\left(t_{r}\right)=0$ and integrating both sides of (44) from 0 to $t_{r}$, one gets

$$
\begin{aligned}
t_{r} & \leq-\int_{s(0)}^{s\left(t_{r}\right)} \frac{d|s|}{\xi\left(|s|+|s|^{\beta}\right)} \\
& =-\left.\frac{1}{\xi(1-\beta)} \ln \left(1+|s|^{1-\beta}\right)\right|_{s\left(t_{0}\right)} ^{s\left(t_{r}\right)} \\
& =-\frac{1}{\xi(1-\beta)} \ln \left(1+|s(0)|^{1-\beta}\right) .
\end{aligned}
$$

Therefore, the state trajectories of the error system (12) will converge to $s(t)=0$ in the finite time $T_{2} \leq-(1 / \varsigma(1-\beta)) \ln (1+$ $\left.|s(0)|^{1-\beta}\right)$.

\section{Numerical Example}

In this section, an example is given to illustrate the effectiveness of the proposed fractional nonsingular terminal fuzzy sliding mode controller in solving the synchronization problem between two fractional-order Genesio-Tesi chaotic systems. 
The mathematical model of an uncertain fractional-order Genesio-Tesi chaotic system, which is chosen as response system with control input and external disturbance, is presented as follows:

$$
D^{\alpha} x(t)=\left\{\begin{array}{l}
D^{\alpha} x_{1}(t)=x_{2} \\
D^{\alpha} x_{2}(t)=x_{3} \\
D^{\alpha} x_{3}(t)=-a x_{1}-b x_{2}-c x_{3}+x_{1}{ }^{2}+\Delta f(x)+d_{x}(t)+u(t),
\end{array}\right.
$$

where

$$
\Delta f(x)+d_{x}(t)=0.2 \cos (2 t) x_{3}-0.3 \sin (t) .
$$

The driving system with an uncertain parameter and an external disturbance is chosen as follows:

$$
\begin{aligned}
& D^{\alpha} y(t) \\
& =\left\{\begin{array}{l}
D^{\alpha} y_{1}(t)=y_{2} \\
D^{\alpha} y_{2}(t)=y_{3} \\
D^{\alpha} y_{3}(t)=-a y_{1}-b y_{2}-c y_{3}+y_{1}{ }^{2}+\Delta g(y)+d_{y}(t),
\end{array}\right.
\end{aligned}
$$

where

$$
\Delta g(y)+d_{y}(t)=0.2 \sin (3 t) y_{3}+0.1 \cos (2 t) .
$$

The above given uncertain parameters and external disturbances $\Delta g(y), \Delta f(x), d_{y}(t)$, and $d_{x}(t)$ are supposed to satisfy Assumptions 2 and 3. In other words, they are assumed to be bounded; meanwhile inequalities (14) and (15) are satisfied. In order to verify Assumptions 2 and 3, we give the simulation results in Figure 1, which shows the boundedness of

$$
\begin{gathered}
\Delta g(y), \\
\Delta f(x), \\
d_{y}(t), \\
d_{x}(t), \\
D^{1-\alpha}(\Delta g(y)-\Delta f(x)), \\
D^{1-\alpha}\left(d_{y}(t)-d_{x}(t)\right), \\
D^{1-\alpha}\left(\Delta g(y)+d_{y}(t)-\Delta f(x)-d_{x}(t)\right) .
\end{gathered}
$$

Now, suppose that $x_{1}(t)$ is in the domain of $[-20,20]$; then the T-S fuzzy model is constructed as follows:

Rule 1: if $x_{1}(t)$ is $M_{1}$, then $D^{\alpha} x(t)=A_{1} x(t)$

Rule 2: if $x_{1}(t)$ is $M_{2}$, then $D^{\alpha} x(t)=A_{2} x(t)$

We obtain the T-S fuzzy models of the reconstructed drive system and the response system in the following:

$$
\begin{aligned}
& D^{\alpha} x(t)=\sum_{i=1}^{2} h_{i}\left[A_{i} x(t)\right]+\Delta \bar{f}(x)+\bar{d}_{x}(t)+U(t) \\
& D^{\alpha} y(t)=\sum_{i=1}^{2} h_{i}\left[A_{i} y(t)\right]+\Delta \bar{g}(y)+\bar{d}_{y}(t),
\end{aligned}
$$

where

$$
\begin{aligned}
& h_{1}(z(t))=\frac{M_{2}-x_{1}}{M_{2}-M_{1}}, \\
& h_{2}(z(t))=\frac{x_{1}-M_{1}}{M_{2}-M_{1}}
\end{aligned}
$$

and $M_{1}$ and $M_{2}$ are the fuzzy sets.

The error dynamic fuzzy system can be obtained from (51) as follows:

$$
\begin{aligned}
D^{\alpha} e(t)= & \sum_{i=1}^{2} h_{i}\left[A_{i} e(t)\right]+\Delta \bar{g}(y)+\bar{d}_{y}(t)-\Delta \bar{f}(x) \\
& -\bar{d}_{x}(t)-U(t),
\end{aligned}
$$

where

$$
\begin{aligned}
& A_{1}=\left[\begin{array}{ccc}
0 & 1 & 0 \\
0 & 0 & 1 \\
M_{1}-a & -b & -c
\end{array}\right], \\
& A_{2}=\left[\begin{array}{ccc}
0 & 1 & 0 \\
0 & 0 & 1 \\
M_{2}-a & -b & -c
\end{array}\right] .
\end{aligned}
$$

Now, choose the related parameters as follows:

$$
\begin{aligned}
\alpha & =0.95, \\
\beta & =0.15, \\
\gamma & =0.5, \\
M_{1} & =-20, \\
M_{2} & =20, \\
a & =1.2, \\
b & =2.92, \\
c & =6, \\
L_{1} & =L_{2}=0.8,
\end{aligned}
$$



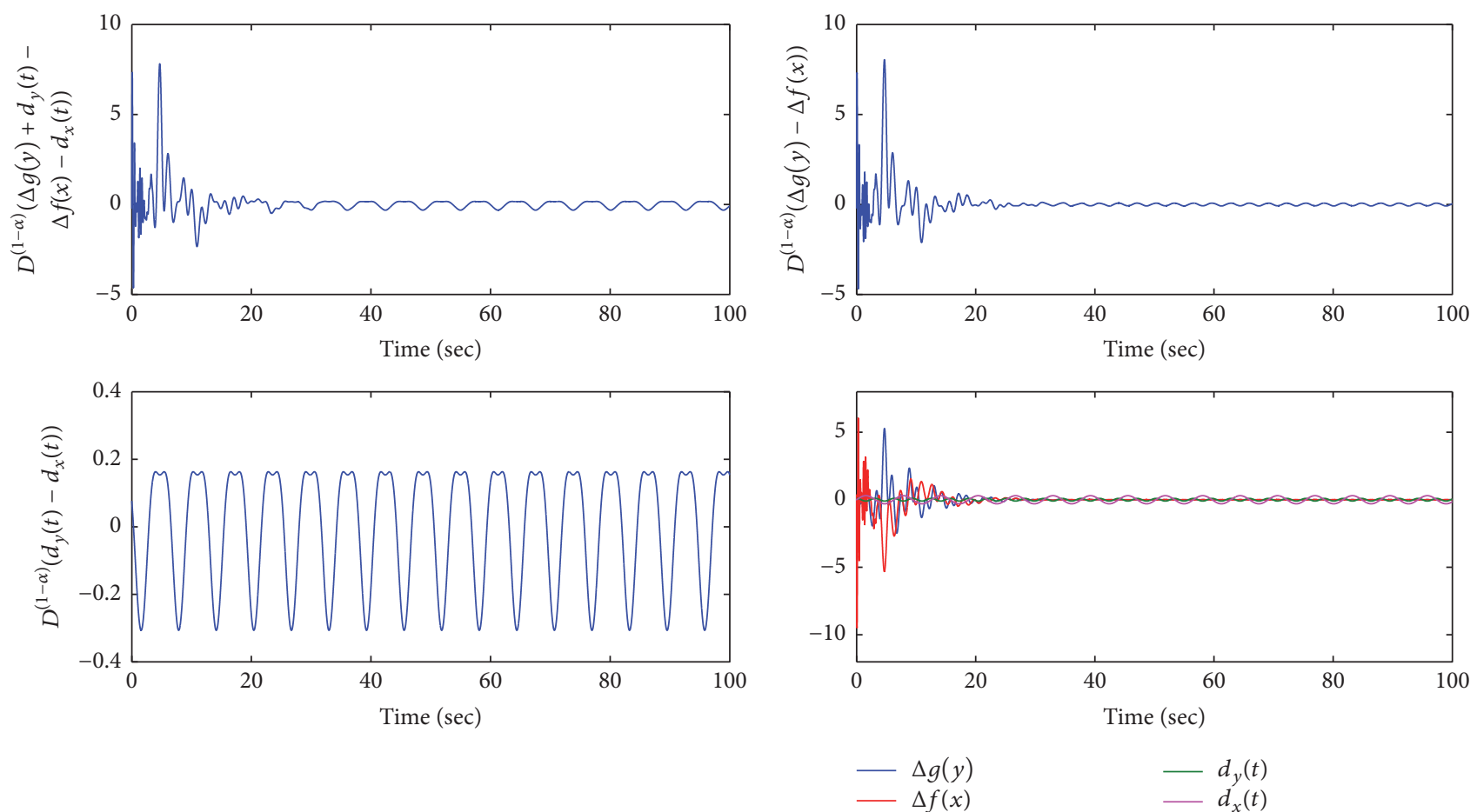

FIGURE 1: The simulation results for the boundedness of the given uncertain parameter and external disturbance.

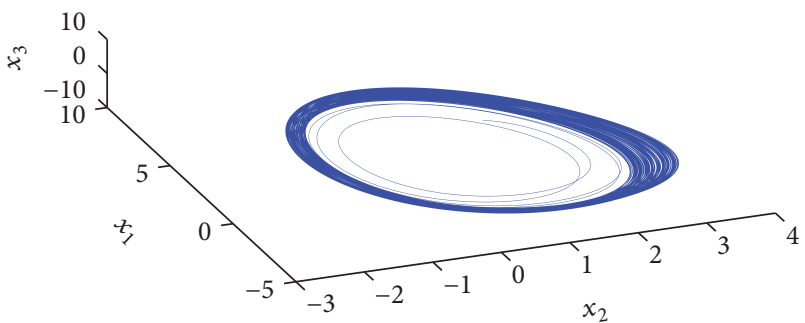

FIGURE 2: The phase trajectory of system (46).

$$
\begin{aligned}
\varsigma_{1} & =2, \\
\varsigma_{2} & =3, \\
\varsigma_{3} & =5, \\
\theta & =0.9, \\
k_{1} & =k_{2}=k_{3}=1
\end{aligned}
$$

when the initial conditions are selected as $\left(x_{1}(0), x_{2}(0)\right.$, $\left.x_{3}(0)\right)=(1,2,2)$ and $\left(y_{1}(0), y_{2}(0), y_{3}(0)\right)=(-0.5,-0.5,1)$. Using the Matlab function about the fractional derivative, fractional integral, and Simulink, we get the following results, which are shown in Figures 2-10.

The phase trajectories of systems (46) and (48) are shown in Figures 2 and 3; from the figures, we can see that there exists chaotic phenomenon in systems (46) and (48) when we set the corresponding parameters as above. Figure 4 shows the uncontrolled state trajectories of systems (46) and (48),



FIGURE 3: The phase trajectory of system (48).

from which we conclude that the uncontrolled systems have chaotic phenomenon. The controlled state trajectories of the drive system (48) and the response system (46) are depicted in Figures 5-7. From the results, we can conclude that the designed fractional-order nonsingular terminal fuzzy sliding mode controller can ensure the asymptotic synchronization between the drive system (48) and the response system (46). The controlled state trajectories of error system $e(t)$ are represented in Figure 8, which can illustrate that the synchronization between systems (48) and (46) can be accomplished in a finite time. Finally, the sliding surface $s(t)$ and control input $u(t)$ are shown in Figures 9 and 10, which verify the effectiveness of the proposed sliding surface and the designed controller.

\section{Conclusions}

In this paper, the fractional-order nonsingular terminal fuzzy sliding mode control scheme has been studied for fractional-order chaotic systems in the presence of 

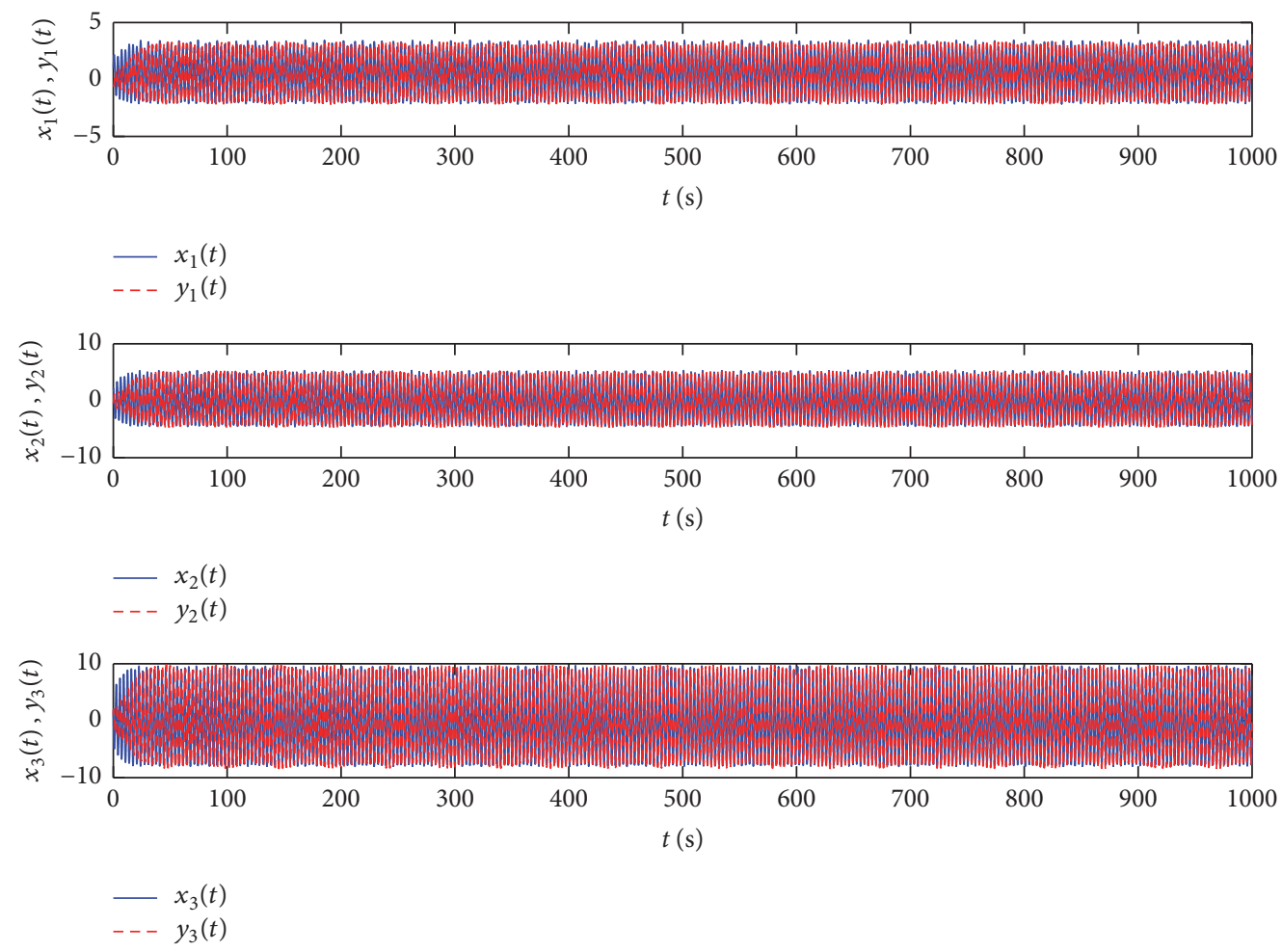

FIgURE 4: The uncontrolled state trajectories of system (46) and system (48).

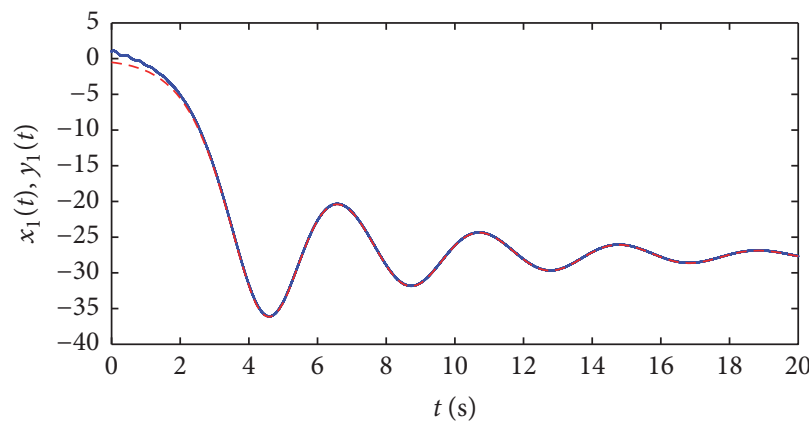

$$
\begin{aligned}
& \longrightarrow x_{1}(t) \\
& ---y_{1}(t)
\end{aligned}
$$

FIGURE 5: The controlled state trajectory of $x_{1}$ and $y_{1}$.



FIgURE 6: The controlled state trajectory of $x_{2}$ and $y_{2}$. 


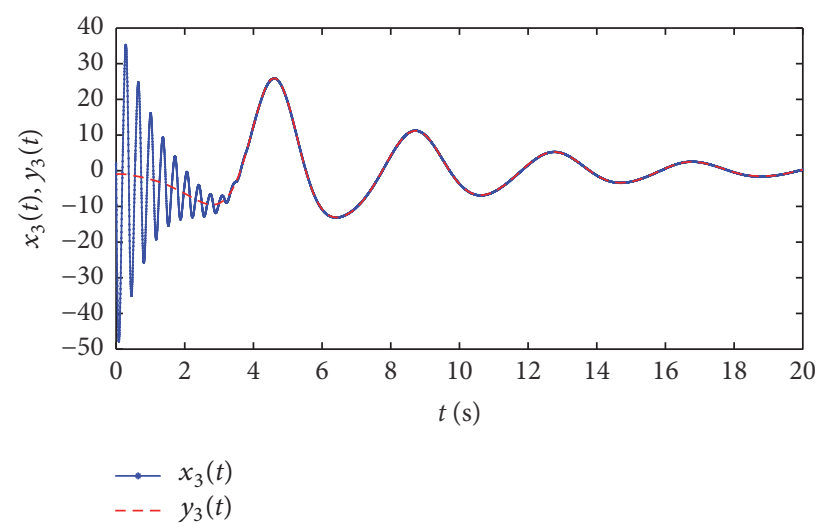

Figure 7: The controlled state trajectory of $x_{3}$ and $y_{3}$.
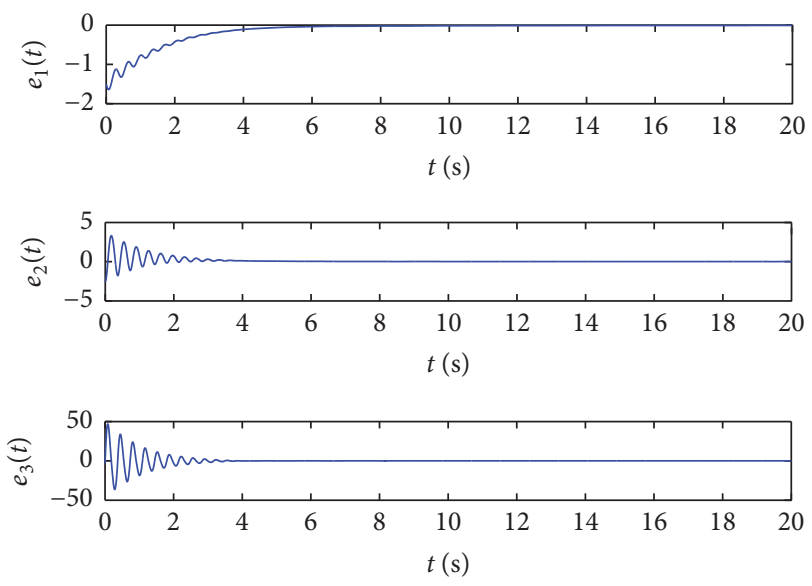

Figure 8: The controlled state trajectory of error system $e(t)$.

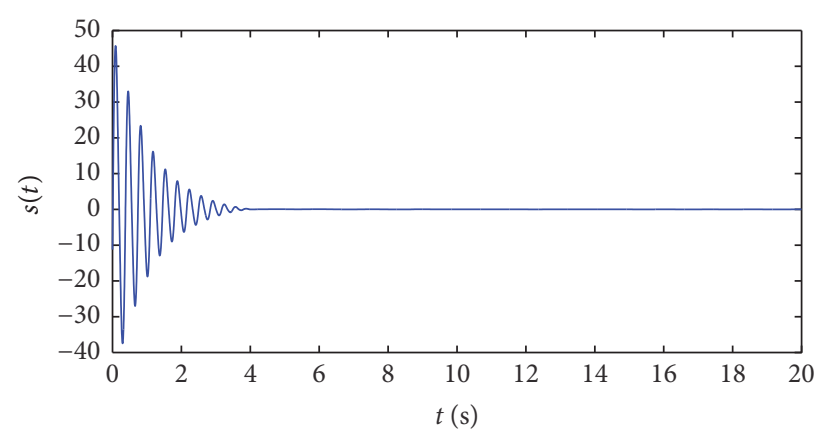

Figure 9: Sliding surface $s(t)$ response with time $t$.

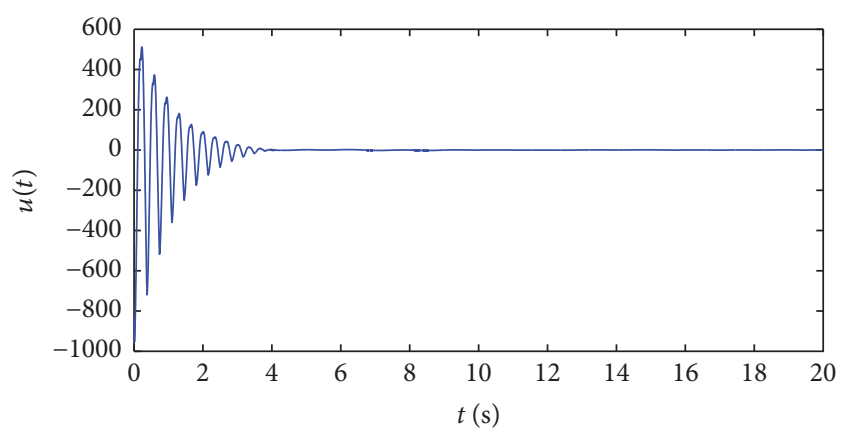

FIgURE 10: The controller $u(t)$ response with time $t$. parameter uncertainty and external disturbances. First, we reconstructed the fractional-order chaotic system based on the T-S fuzzy model. Then, we proposed a new fractionalorder terminal sliding surface that has the finite-time stability characteristics. Based on the sliding mode control theory and fractional Lyapunov stability theory, a fuzzy sliding mode control law is designed to ensure the occurrence of the sliding motion in a finite time. The numerical simulations show the effectiveness of the proposed controller.

\section{Conflicts of Interest}

The authors declare that there are no conflicts of interest regarding the publication of this paper.

\section{Acknowledgments}

This paper is supported by the National Natural Science Foundation of China (nos. U1604146, U1404610, 61473115, and 61203047), Science and Technology Research Project in Henan Province (nos. 152102210273 and 162102410024), and Foundation for the University Technological Innovative Talents of Henan Province (no. 18HASTIT019). The first author would like to sincerely thank Professor Om P. Agrawal, Department of Mechanical Engineering and Energy Processes, Southern Illinois University, Carbondale, IL, USA, for reading this paper and providing extensive feedback. The first author would also like to thank Professor R. Koc, Chair, Department of Mechanical Engineering and Energy Processes, Southern Illinois University, Carbondale, IL, USA, for hosting her during the period from 8 April 2016 to 8 April 2017.

\section{References}

[1] I. Podlubny, I. Petráš, B. M. Vinagre, P. O’Leary, and L. Dorčák, "Analogue realizations of fractional-order controllers," Nonlinear Dynamics, vol. 29, no. 1-4, pp. 281-296, 2002.

[2] O. P. Agrawal, "Solution for a fractional diffusion-wave equation defined in a bounded domain," Nonlinear Dynamics, vol. 29, no. 1-4, pp. 145-155, 2002. 
[3] K. Diethelm, N. J. Ford, and A. D. Freed, "A predictor-corrector approach for the numerical solution of fractional differential equations," Nonlinear Dynamics, vol. 29, no. 1-4, pp. 3-22, 2002.

[4] Y. Luo, Y. Chen, and Y. Pi, "Experimental study of fractional order proportional derivative controller synthesis for fractional order systems," Mechatronics, vol. 21, no. 1, pp. 204-214, 2011.

[5] M. P. Aghababa, "Chaos in a fractional-order micro-electromechanical resonator and its suppression," Chinese Physics B, vol. 21, no. 10, Article ID 100505, 2012.

[6] I. Grigorenko and E. Grigorenko, "Chaotic dynamics of the fractional Lorenz system," Physical Review Letters, vol. 91, Article ID 034101, 2003.

[7] A. S. Hegazi, E. Ahmed, and A. E. Matouk, "On chaos control and synchronization of the commensurate fractional order Liu system," Communications in Nonlinear Science and Numerical Simulation, vol. 18, no. 5, pp. 1193-1202, 2013.

[8] X. Wu, H. Lu, and S. Shen, "Synchronization of a new fractionalorder hyperchaotic system," Physics Letters. A, vol. 373, no. 2728, pp. 2329-2337, 2009.

[9] J. Yuan, B. Shi, and W. Ji, "Adaptive sliding mode control of a novel class of fractional chaotic systems," Advances in Mathematical Physics, vol. 2013, Article ID 576709, 13 pages, 2013.

[10] D. Matignon, "Stability results for fractional differential equations with applications to control processing," in Proceedings of the IMACS, vol. 1996, pp. 963-968, IEEE-SMC, Lille, France.

[11] A. Kiani-B, K. Fallahi, N. Pariz, and H. Leung, "A chaotic secure communication scheme using fractional chaotic systems based on an extended fractional Kalman filter," Communications in Nonlinear Science and Numerical Simulation, vol. 14, no. 3, pp. 863-879, 2009.

[12] S. K. Agrawal, M. Srivastava, and S. Das, "Synchronization of fractional order chaotic systems using active control method," Chaos, Solitons \& Fractals, vol. 45, no. 6, pp. 737-752, 2012.

[13] X. Wang, X. Zhang, and C. Ma, "Modified projective synchronization of fractional-order chaotic systems via active sliding mode control," Nonlinear Dynamics, vol. 69, no. 1-2, pp. 511-517, 2012.

[14] L. Y. T. Andrew, L. Xian-Feng, C. Yan-Dong, and Z. Hui, "A novel adaptive-impulsive synchronization of fractional-order chaotic systems," Chinese Physics B, vol. 24, no. 10, Article ID 100502, 2015.

[15] A. Bouzeriba, A. Boulkroune, and T. Bouden, "Fuzzy adaptive synchronization of uncertain fractional-order chaotic systems," International Journal of Machine Learning and Cybernetics, vol. 7, no. 5, pp. 893-908, 2016.

[16] G. Peng, Y. Jiang, and F. Chen, "Generalized projective synchronization of fractional order chaotic systems," Physica A: Statistical Mechanics and Its Applications, vol. 387, no. 14, pp. 3738-3746, 2008.

[17] V. I. Utkin, Sliding Modes in Control and Optimization, Springer, Berlin, Germany, 1992.

[18] F. Li, L. Wu, P. Shi, and C.-C. Lim, "State estimation and sliding mode control for semi-Markovian jump systems with mismatched uncertainties," Automatica, vol. 51, pp. 385-393, 2015.

[19] H. Li, P. Shi, D. Yao, and L. Wu, "Observer-based adaptive sliding mode control for nonlinear Markovian jump systems," Automatica, vol. 64, pp. 133-142, 2016.

[20] L. Wu, W. X. Zheng, and H. Gao, "Dissipativity-based sliding mode control of switched stochastic systems," IEEE Transactions on Automatic Control, vol. 58, no. 3, pp. 785-791, 2013.
[21] M. S. Tavazoei and M. Haeri, "Synchronization of chaotic fractional-order systems via active sliding mode controller," Physica A: Statistical Mechanics and Its Applications, vol. 387, no. 1, pp. 57-70, 2008.

[22] C. Yin, S.-M. Zhong, and W.-F. Chen, "Design of sliding mode controller for a class of fractional-order chaotic systems," Communications in Nonlinear Science and Numerical Simulation, vol. 17, no. 1, pp. 356-366, 2012.

[23] D.-Y. Chen, Y.-X. Liu, X.-Y. Ma, and R.-F. Zhang, "Control of a class of fractional-order chaotic systems via sliding mode," Nonlinear Dynamics, vol. 67, no. 1, pp. 893-901, 2012.

[24] N. Yang and C. Liu, "A novel fractional-order hyperchaotic system stabilization via fractional sliding-mode control," Nonlinear Dynamics, vol. 74, no. 3, pp. 721-732, 2013.

[25] J. Yang, S. Li, J. Su, and X. Yu, "Continuous nonsingular terminal sliding mode control for systems with mismatched disturbances," Automatica, vol. 49, no. 7, pp. 2287-2291, 2013.

[26] M. P. Aghababa, "Finite-time chaos control and synchronization of fractional-order nonautonomous chaotic (hyperchaotic) systems using fractional nonsingular terminal sliding mode technique," Nonlinear Dynamics, vol. 69, no. 1-2, pp. 247-261, 2012.

[27] M. P. Aghababa, "A novel terminal sliding mode controller for a class of non-autonomous fractional-order systems," Nonlinear Dynamics. An International Journal of Nonlinear Dynamics and Chaos in Engineering Systems, vol. 73, no. 1-2, pp. 679-688, 2013.

[28] M. P. Aghababa, "No-chatter variable structure control for fractional nonlinear complex systems," Nonlinear Dynamics, vol. 73, no. 4, pp. 2329-2342, 2013.

[29] X. Yang, Q. Song, Y. Liu, and Z. Zhao, "Finite-time stability analysis of fractional-order neural networks with delay," Neurocomputing, vol. 152, pp. 19-26, 2015.

[30] L. Chen, W. Pan, R. Wu et al., "New result on finite-time stability of fractional-order nonlinear delayed systems," Journal of Computational and Nonlinear Dynamics, vol. 10, no. 6, 2015.

[31] B. Xin and J. Zhang, "Finite-time stabilizing a fractional-order chaotic financial system with market confidence," Nonlinear Dynamics, vol. 79, no. 2, pp. 1399-1409, 2015.

[32] C. Li and J. Zhang, "Synchronisation of a fractional-order chaotic system using finite-time input-to-state stability," International Journal of Systems Science, vol. 47, no. 10, pp. 2440 2448, 2016.

[33] M. P. Aghababa, "Synchronization and stabilization of fractional second-order nonlinear complex systems," Nonlinear Dynamics, vol. 80, no. 4, pp. 1731-1744, 2015.

[34] H. Wang, Z.-Z. Han, Q.-Y. Xie, and W. Zhang, "Finite-time chaos control via nonsingular terminal sliding mode control," Communications in Nonlinear Science \& Numerical Simulation, vol. 14, no. 6, pp. 2728-2733, 2009.

[35] M. P. Aghababa, "A fractional sliding mode for finite-time control scheme with application to stabilization of electrostatic and electromechanical transducers," Applied Mathematical Modelling, vol. 39, no. 20, pp. 6103-6113, 2015.

[36] I. Podlubny, Fractional Differential Equations, Academic Press, New York, NY, USA, 1999. 


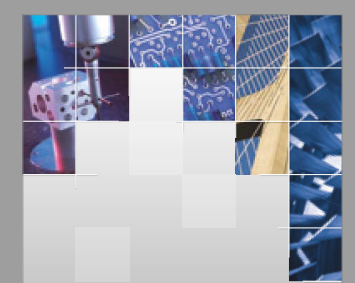

\section{Enfincering}
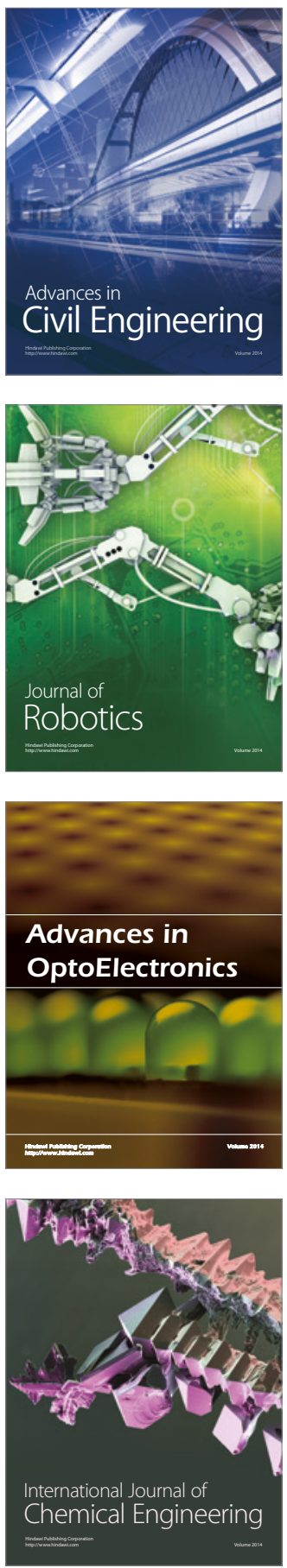

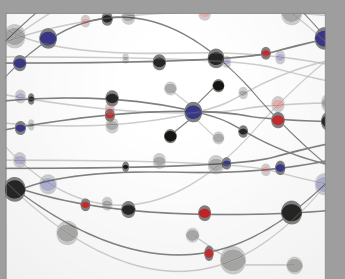

The Scientific World Journal

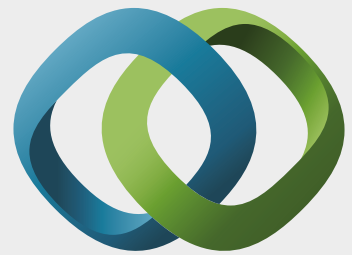

\section{Hindawi}

Submit your manuscripts at

https://www.hindawi.com
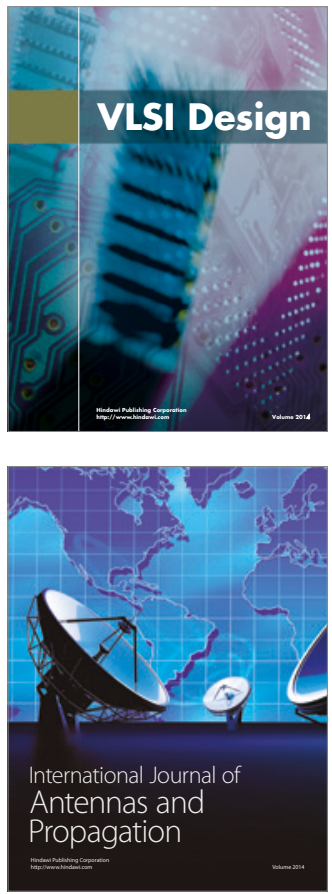

\section{Rotating}

Machinery

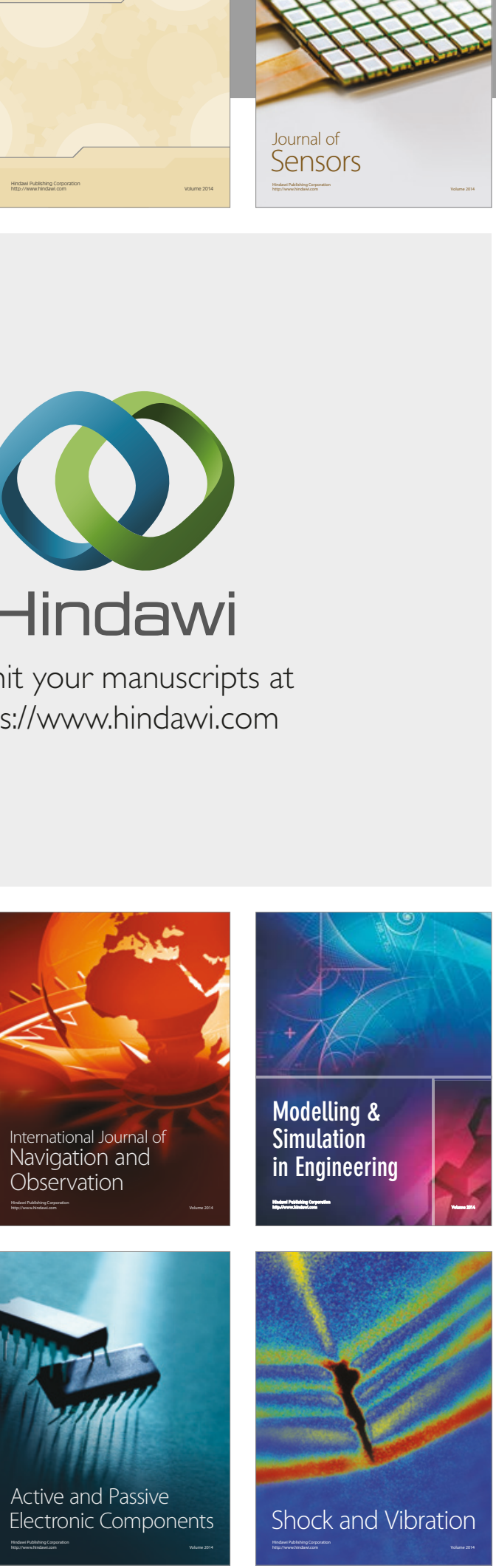
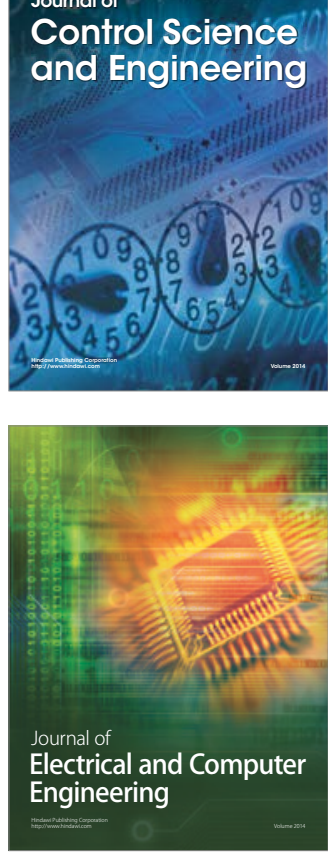

Distributed

Journal of

Control Science

and Engineering
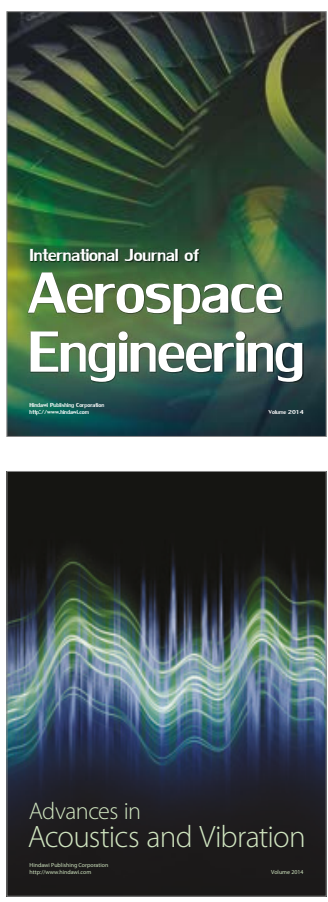

Sensor Networks 